\title{
Cloud Service under the Presence of Privacy Concern
}

\author{
Eunjin Kim* \\ Department of International Industrial Information, Kyonggi University, Suwon, Republic of Korea
}

Received: 26 Sep. 2013, Revised: 24 Dec. 2013, Accepted: 25 Dec. 2013

Published online: 1 Sep. 2014

\begin{abstract}
Privacy is an important issue for cloud computing in terms of user trust. Hence, it needs to be considered in its service design. In this study, we investigate the impact of privacy concern on cloud service design in terms of collecting and storing information of users. With an analytical model, we show that, in the presence of privacy concern of users and cloud service firms' difference in their service ability in utilizing users' information for their cloud service, a firm that possesses inferior service ability might choose to demand and require users to input and store user information that include highly private ones, while a firm with superior service ability demands less. An interesting point is that, such competing firms' choosing different privacy policies can further reduce competition between cloud service firms.
\end{abstract}

Keywords: Privacy concern, Cloud computing, Service quality

\section{Introduction}

As cloud services store and process users' data on machines that the users do not own or operate, this lessen users' control on their data and information and further introduces privacy issue. Privacy issue is hence central to user concerns about adoption of cloud computing and is a key challenge for cloud service firms when designing their service. It is known that users value privacy[1,2]. However, this does not mean that users are uniform in their concern for privacy. Previous studies have shown that the willingness to share information is known to be related to a user's perception of risks of disclosure[3], and thus users vary in their concerns for privacy[4,5]. That is, different users have different interests and views about the amount of information to disclose during a certain transaction with the firm[1].

In this study, we investigate the impact of users' privacy concern on cloud service design in terms of collecting and storing information of users. With an analytical model, we show that, in the presence of users' heterogeneity in their concern for privacy and firms' difference in their service ability in utilizing users' information for their cloud service, a firm with inferior ability might choose to design their service to demand information that includes highly privacy sensitive ones, while a firm with superior ability chooses to demands less. An interesting point is that, through making firms to choose different strategy in their service design for privacy and to target users of different level of privacy concerns, user heterogeneity in information privacy concern can reduce competition between cloud service firms. The rest of the paper is organized as follows. In Section 2, we describe the model. We then analyze the model and discuss our findings in Section 3. We conclude our research in the final section.

\section{The Model}

\subsection{Assumption of the model}

We develop a two-stage game of duopoly firms. In the first stage, firms decide the level of users' information they will collect and store for cloud service. As firms can collect and store more private information from users, they can serve users with better and more sophisticated cloud services with utilizing the information. That is, the level of users' information collected and stored determines the quality of cloud service that firms can provide to users. In the second stage, firms engage in price competition. Our model is as follows.

It is assumed that the quality of cloud service is determined by both the level of information collected and the ability of firms to utilize such information. Therefore,

\footnotetext{
*Corresponding author e-mail: ejkim777@kgu.ac.kr
} 
the quality of cloud service of firm is

$$
q_{i}=s_{i} x_{i}
$$

Here, $q_{i}, s_{i}$, and $x_{i}$ represent the quality of cloud service of a firm, the ability of a firm to utilize users' information for the service, and the level of users' information collected, respectively. Here, we assume that $s_{i}$ is exogenously given and $x_{i}$ is endogenously determined by firms' decisions.

We assume that Firms 1 and 2 differ in their ability to utilize information. Firm 1 is assumed to be superior to Firm 2 in this respect. Mathematically, $s_{2}=\lambda s_{1}$ where $\lambda<1$. Here, a smaller $\lambda$ represents a greater gap between the two competitors in terms of this ability. Since the focus of our analysis is the ability gap between duopolies, we assume $s_{1}=1$ for simplicity of analysis.

Concerning users' heterogeneity in their concern for privacy, a previous study divided users largely into two types, convenience seekers who try to seek high service benefits from giving up privacy while possessing low concern about privacy and privacy seekers who are privacy sensitive[5]. Likewise, we assume two types of users: convenience seekers and privacy seekers. Privacy seekers are those who are privacy sensitive and intend to share only basic information to get cloud service, and are not willing to share highly private information with firms because of the concerns about privacy. Unlike privacy seekers, convenience seekers are not sensitive to privacy and share not only basic information but also intimate private information with firms. The total number of users in the market is normalized as 1 where $\alpha$ is the portion of convenience seekers and $(1-\alpha)$ is the portion of privacy seekers. If a firm demands basic information only, then the firm can attract both types of users. However, if the firm requests additional private information beyond basic information, the firm can attract only convenience seekers. For simplicity, basic information is assumed to be low-level information $\left(x_{L}=1\right)$, and information that includes both basic and additional information is assumed to be high-level information $\left(x_{H}=2\right)$. In addition to this heterogeneity in information privacy concerns, users differ in their tastes for cloud service quality, described by the parameter $\theta$, which is uniformly distributed on the interval $[0,1]$.

In the first stage, firms simultaneously choose the level of information that they will demand of users, $x_{i}$. In the second stage of competition, the firms set their prices $p_{i}$. We derive the subgame-perfect Nash equilibrium strategies in stage 1 by first examining Nash equilibrium strategies in stage 2 .

\section{Analysis and Results}

\subsection{Pricing decision (Stage 2)}

After stage 1, there are four possible subgames: (i) $x_{1}=x_{H}$ and $x_{2}=x_{L}$ (i.e., Firm 1 chooses $x_{H}$, and Firm 2 chooses $x_{L}$ in stage 1), (ii) $x_{1}=x_{H}$ and $x_{2}=x_{H}$, (iii) $x_{1}=x_{L}$ and $x_{2}=$ $x_{L}$, (iv) $x_{1}=x_{L}$ and $x_{2}=x_{H}$. We analyze each subgame by finding the Nash equilibrium prices and profits.

Subgame 1: Firm 1's $x_{1}=x_{H}$ and Firm 2's $x_{2}=x_{L}$

When $x_{1}=x_{H}$ and $x_{2}=x_{L}$, Firm 1 can attract only convenience seekers, while Firm 2 can attract both types of users. From the utility function of users, we can derive the demand for Firm $1 d_{1}$ and for Firm $2 d_{2}$ as follows. (In deriving demand functions, we only consider the conditions under which both firms have positive market shares, since no firm will choose to be wiped out.)

$$
\begin{aligned}
& d_{1}=\alpha\left(1-\frac{p_{1}-p_{2}}{s_{1} x_{H}-s_{2} x_{L}}\right), \text { and } \\
& d_{2}=\alpha\left(\frac{p_{1}-p_{2}}{s_{1} x_{H}-s_{2} x_{L}}-\frac{p_{2}}{s_{2} x_{L}}\right)+(1-\alpha)\left(1-\frac{p_{2}}{s_{2} x_{L}}\right) \\
& \text { if } \frac{p_{2}}{s_{2} x_{L}} \leq \frac{p_{1}}{s_{1} x_{H}}<1, \\
& d_{1}=\alpha\left(1-\frac{p_{1}}{s_{1} x_{H}}\right), \text { and } d_{2}=(1-\alpha)\left(1-\frac{p_{2}}{s_{2} x_{L}}\right) \\
& \text { if } \frac{p_{1}}{s_{1} x_{H}}<\frac{p_{2}}{s_{2} x_{L}}<1 .
\end{aligned}
$$

The profits of Firms 1 and 2 are $\pi_{1}=d_{1} p_{1}$ and $\pi_{2}=d_{2} p_{2}$, respectively. From the first- and second-order conditions $\left(\delta \pi_{i} / \delta p_{i}=0\right.$, and $\left.\delta^{2} \pi_{i} / \delta p_{i}^{2}<0\right)$ and given our assumption for $s_{1}, x_{L}$ and $x_{H}$, we can derive the best response functions of Firms 1 and 2 as follows.

$$
\begin{gathered}
\mathbf{p}_{\mathbf{1}}=\left(\begin{array}{cl}
\frac{p_{2}}{2}-\frac{\lambda}{2}+1 & \text { if } p_{2} \leq \frac{\lambda(2-\lambda)}{(4-\lambda)} \\
\frac{2 p_{2}}{\lambda} & \text { if } \frac{\lambda(2-\lambda)}{(4-\lambda)}<p_{2} \leq \frac{\lambda}{2} \\
1 & \text { if } p_{2}>\frac{\lambda}{2}
\end{array}\right), \text { and } \\
\mathbf{p}_{2}=\left(\begin{array}{cc}
\frac{\lambda}{2} & \text { if } p_{1} \leq \hat{p}_{1} \\
\frac{\alpha \lambda p_{1}+2 \lambda-2 \alpha \lambda-\lambda^{2}+\alpha \lambda^{2}}{2 \alpha \lambda-2 \lambda+4} & \text { if } p_{1}>\hat{p}_{1}
\end{array}\right)
\end{gathered}
$$

where

$\hat{p_{1}}=(2 \alpha+\lambda-\alpha \lambda-2+\sqrt{(1-\alpha)(2-\lambda)(\alpha \lambda-\lambda+2)}) / \alpha$. Based on the response functions, we can derive the equilibrium prices of firms as

$$
\begin{gathered}
p_{1}=\frac{\alpha \lambda-\lambda-2 \alpha+2}{\alpha \lambda-\lambda-\alpha+2}, \text { and } \\
p_{2}=\frac{2 \lambda-2 \alpha \lambda-\lambda^{2}+\alpha \lambda^{2}}{2 \alpha \lambda-2 \lambda-2 \alpha+4} \text { when } \alpha \leq \frac{\lambda}{\lambda+2}, \text { and } \\
p_{1}=\frac{2 \alpha \lambda-6 \lambda+\lambda^{2}-\alpha \lambda^{2}+8}{3 \alpha \lambda-4 \lambda+8}, \text { and } \\
p_{2}=\frac{4 \lambda-2 \alpha \lambda-2 \lambda^{2}+\alpha \lambda^{2}}{3 \alpha \lambda-4 \lambda+8} \text { when } \alpha>\frac{\lambda}{\lambda+2} .
\end{gathered}
$$

From the equilibrium prices, we can derive equilibrium profits. The equilibrium profits of Firms 1 
and 2 when $\alpha \leq \lambda /(\lambda+2)$ are derived from inserting the prices of (2) into the profit functions. We denote these equilibrium profits as $\pi_{1 \mid\left(x_{H}, x_{L}\right)}=\dot{\pi}_{1 \mid\left(x_{H}, x_{L}\right)}$ and $\pi_{2 \mid\left(x_{H}, x_{L}\right)}=\dot{\pi}_{2 \mid\left(x_{H}, x_{L}\right)}$. Likewise, plugging the equilibrium prices of (3) into the profit functions, the equilibrium profits of Firms 1 and 2 when $\alpha>\lambda /(\lambda+2)$ are derived as $\pi_{1 \mid\left(x_{H}, x_{L}\right)}=\ddot{\pi}_{1 \mid\left(x_{H}, x_{L}\right)}$ and $\pi_{2 \mid\left(x_{H}, x_{L}\right)}=\ddot{\pi}_{2 \mid\left(x_{H}, x_{L}\right)}$.

Subgame 2: Firm 1's $x_{1}=x_{H}$ and Firm 2's $x_{2}=x_{H}$

When $x_{1}=x_{H}$ and $x_{2}=x_{H}$, both Firm 1 and Firm 2 attract only convenience seekers. We can derive the demand for Firms 1 and 2 as

$$
\begin{aligned}
& d_{1}=\alpha\left(1-\frac{p_{1}-p_{2}}{s_{1} x_{H}-s_{2} x_{H}}\right), \text { and } \\
& d_{2}=\alpha\left(\frac{p_{1}-p_{2}}{s_{1} x_{H}-s_{2} x_{H}}-\frac{p_{2}}{s_{2} x_{H}}\right) \text { if } \frac{p_{2}}{s_{2} x_{H}} \leq \frac{p_{1}}{s_{1} x_{H}}<1 .
\end{aligned}
$$

It can be shown that the equilibrium prices are

$$
p_{1}=\frac{4(1-\lambda)}{4-\lambda}, \text { and } p_{2}=\frac{2 \lambda(1-\lambda)}{4-\lambda} .
$$

We denote the equilibrium prices as $\pi_{1 \mid\left(x_{H}, x_{H}\right)}$ and $\pi_{2 \mid\left(x_{H}, x_{H}\right)}$.

Subgame 3: Firm 1's $x_{1}=x_{L}$ and Firm 2's $x_{2}=x_{L}$

Firms 1 and 2 can attract both types of users. The demand for Firms 1 and 2 is

$$
\begin{aligned}
& d_{1}=1-\frac{p_{1}-p_{2}}{s_{1} x_{L}-s_{2} x_{L}}, \text { and } d_{2}=\frac{p_{1}-p_{2}}{s_{1} x_{L}-s_{2} x_{L}}-\frac{p_{2}}{s_{2} x_{L}} \\
& \text { if } \frac{p_{2}}{s_{2} x_{L}} \leq \frac{p_{1}}{s_{1} x_{L}}<1 .
\end{aligned}
$$

It can be shown that the equilibrium prices are

$$
p_{1}=\frac{2(1-\lambda)}{4-\lambda}, \text { and } p_{2}=\frac{\lambda(1-\lambda)}{4-\lambda} .
$$

We donote the equilibrium prices as $\pi_{1 \mid\left(x_{L}, x_{L}\right)}$ and $\pi_{2 \mid\left(x_{L}, x_{L}\right)}$.

Subgame 4: Firm 1's $x_{1}=x_{L}$ and Firm 2's $x_{2}=x_{H}$

When $x_{1}=x_{L}$ and $x_{2}=x_{H}$, Firm 1 attracts both types of users. However, Firm 2 can serve only convenience seekers. When $\lambda \leq x_{L} / x_{H}$, the demand functions are

$$
\begin{gathered}
d_{1}=\alpha\left(1-\frac{p_{1}-p_{2}}{s_{1} x_{L}-s_{2} x_{H}}\right)+(1-\alpha)\left(1-\frac{p_{1}}{s_{1} x_{L}}\right) \\
\text { and } d_{2}=\alpha\left(\frac{p_{1}-p_{2}}{s_{1} x_{L}-s_{2} x_{H}}-\frac{p_{2}}{s_{2} x_{H}}\right) \text { if } \frac{p_{2}}{s_{2} x_{H}} \leq \frac{p_{1}}{s_{1} x_{L}}<1 \\
d_{1}=(1-\alpha)\left(1-\frac{p_{1}}{s_{1} x_{L}}\right), \text { and } \\
d_{2}=\alpha\left(1-\frac{p_{2}}{s_{2} x_{H}}\right) \text { if } \frac{p_{2}}{s_{2} x_{H}}<1 \leq \frac{p_{1}}{s_{1} x_{L}} .
\end{gathered}
$$

We can derive the best response functions of Firms 1 and 2 as follows.

$$
\mathbf{p}_{\mathbf{1}}=\left(\begin{array}{cc}
\frac{1}{2} & \text { if } p_{2} \leq \hat{p}_{2} \\
\frac{\alpha p_{2}-2 \lambda+1}{4 \alpha \lambda-4 \lambda+2} & \text { if } p_{2}>\hat{p}_{2}
\end{array}\right), \text { and }
$$

$$
\mathbf{p}_{2}=\left(\begin{array}{cl}
\lambda p_{1} & \text { if } p_{1} \leq \frac{1-2 \lambda}{1-\lambda} \\
p_{1}+2 \lambda-1 & \text { if } \frac{1-2 \lambda}{1-\lambda}<p_{1} \leq 1-\lambda \\
\lambda & \text { if } p_{1}>1-\lambda
\end{array}\right)
$$

where

$\hat{p_{2}}=(2 \lambda-1+\sqrt{(1-\alpha)(1-2 \lambda)(2 \alpha \lambda-2 \lambda+1)}) / \alpha$.

Based on the best response functions, we can derive the equilibrium prices of both firms as

$$
\begin{aligned}
& p_{1}=\frac{2 \alpha \lambda-2 \lambda-\alpha+1}{4 \alpha \lambda-4 \lambda-\alpha+2}, \text { and } \\
& p_{2}=\frac{6 \lambda-4 \alpha \lambda-8 \lambda^{2}+8 \alpha \lambda^{2}-1}{4 \alpha \lambda-4 \lambda-\alpha+2} \\
& \text { when } \alpha \leq \frac{3 \lambda-1}{3 \lambda},
\end{aligned}
$$

and

$$
\begin{aligned}
& p_{1}=\frac{1-2 \lambda}{3 \alpha \lambda-4 \lambda+2}, \text { and } p_{2}=\frac{\lambda-2 \lambda^{2}}{3 \alpha \lambda-4 \lambda+2} \\
& \text { when } \alpha>\frac{3 \lambda-1}{3 \lambda} .
\end{aligned}
$$

We denote equilibrium profits when $\lambda \leq x_{L} / x_{H}$ and $\alpha \leq(3 \lambda-1) / 3 \lambda$ as $\dot{\pi}_{1 \mid\left(x_{L}, x_{H}\right)}$ and $\dot{\pi}_{2 \mid\left(x_{L}, x_{H}\right)}$. Equilibrium profits when $\alpha>(3 \lambda-1) / 3 \lambda$ are $\ddot{\pi}_{1 \mid\left(x_{L}, x_{H}\right)}$ and $\ddot{\pi}_{2 \mid\left(x_{L}, x_{H}\right)}$.

In the case when $\lambda>x_{L} / x_{H}$, the demand functions are

$$
\begin{aligned}
d_{1} & =\alpha\left(\frac{p_{2}-p_{1}}{s_{2} x_{H}-s_{1} x_{L}}-\frac{p_{1}}{s_{1} x_{L}}\right)+(1-\alpha)\left(1-\frac{p_{1}}{s_{1} x_{L}}\right), \text { and } \\
d_{2}=\alpha & \alpha\left(1-\frac{p_{2}-p_{1}}{s_{2} x_{H}-s_{1} x_{L}}\right) \text { if } \frac{p_{1}}{s_{1} x_{L}} \leq \frac{p_{2}}{s_{2} x_{H}}<1, \\
d_{1} & =(1-\alpha)\left(1-\frac{p_{1}}{s_{1} x_{L}}\right), \text { and } d_{2}=\alpha\left(1-\frac{p_{2}}{s_{2} x_{H}}\right) \text { if } \\
\frac{p_{2}}{s_{2} x_{H}} & <\frac{p_{1}}{s_{1} x_{L}}<1 .
\end{aligned}
$$

The best response functions of Firms 1 and 2 are as follows.

$$
\begin{gathered}
\mathbf{p}_{\mathbf{1}}=\left(\begin{array}{cc}
\frac{1}{2} & \text { if } p_{2} \leq \tilde{p}_{2} \\
\frac{\alpha p_{2}+\alpha+2 \lambda-2 \alpha \lambda-1}{2 \alpha+4 \lambda-2} & \text { if } p_{2}>\tilde{p}_{2}
\end{array}\right), \text { and } \\
\mathbf{p}_{2}=\left(\begin{array}{cl}
\frac{p_{1}}{2}+\lambda-\frac{1}{2} & \text { if } p_{1}<\frac{2 \lambda-1}{4 \lambda-1} \\
2 \lambda p_{1} & \text { if } \frac{2 \lambda-1}{4 \lambda-1}<p_{1} \leq \frac{1}{2} \\
\lambda & \text { if } p_{1}>\frac{1}{2}
\end{array}\right)
\end{gathered}
$$

where

$\tilde{p}_{2}=(2 \alpha \lambda-2 \lambda-\alpha+1+\sqrt{(1-\alpha)(1-2 \lambda)(1-2 \lambda+\alpha)}) / \alpha$. Based on the best response functions, we can derive the equilibrium prices of both firms as 


$$
\begin{aligned}
& p_{1}=\frac{2 \alpha \lambda-2 \lambda-\alpha+1}{2 \alpha \lambda-4 \lambda-2 \alpha+2}, \text { and } \\
& p_{2}=\frac{\lambda-\alpha \lambda-2 \lambda^{2}+2 \alpha \lambda^{2}}{\alpha \lambda-2 \lambda-\alpha+1} \text { when } \alpha \leq \frac{1}{2 \lambda+1},
\end{aligned}
$$

and

$$
\begin{aligned}
& p_{1}=\frac{\alpha+4 \lambda-2 \alpha \lambda-2}{3 \alpha+8 \lambda-4}, \text { and } \\
& p_{2}=\frac{2 \alpha \lambda-6 \lambda-\alpha+8 \lambda^{2}+1}{3 \alpha+8 \lambda-4} \text { when } \alpha>\frac{1}{2 \lambda+1} .
\end{aligned}
$$

The equilibrium profits when $\lambda>x_{L} / x_{H}$ and $\alpha \leq 1 /(2 \lambda+1)$ are derived from plugging the prices from (8) into the profit functions, which are $\pi_{1 \mid\left(x_{L}, x_{H}\right)}=\dddot{\pi}_{1 \mid\left(x_{L}, x_{H}\right)}$ and $\pi_{2 \mid\left(x_{L}, x_{H}\right)}=\dddot{\pi}_{2 \mid\left(x_{L}, x_{H}\right)}$. The equilibrium profits when $\alpha>(3 \lambda-1) / 3 \lambda$ are $\pi_{1 \mid\left(x_{L}, x_{H}\right)}=\dddot{\pi}_{1 \mid\left(x_{L}, x_{H}\right)}$ and $\pi_{2 \mid\left(x_{L}, x_{H}\right)}=\dddot{\pi}_{2 \mid\left(x_{L}, x_{H}\right)}$.

\subsection{Required information-level decisions(Stage 1)}

In the first stage, firms simultaneously choose the level of information that they will demand of users, $x_{i}$. From analyzing equilibriums, we can get the following propositions that show the effect of a high level of privacy concern on restrictions against firms' collecting and using user information and on firms' profit.

Proposition 1. When most users are privacy seekers (i.e., when the portion of convenience seekers is in the range of $\alpha<\bar{\alpha}$ ), both firms choose to only demand basic information $\left(x_{L}\right)$ from users to serve both types of users including privacy seekers and convenience seekers. That is, $\left(x_{L}, x_{L}\right)$ is the unique subgame-perfect Nash equilibrium under this condition.

Proof. If $\alpha<\alpha_{a}, \pi_{1 \mid\left(x_{L}, x_{L}\right)}>\pi_{1 \mid\left(x_{H}, x_{L}\right)}$. Therefore, Firm 1 's best response to Firm 2's choosing $x_{L}$ is $x_{L}$ if $\alpha<\alpha_{a}$. If $\alpha<\alpha_{b}, \pi_{1 \mid\left(x_{L}, x_{H}\right)}>\pi_{1 \mid\left(x_{H}, x_{H}\right)}$. Therefore, Firm 1 chooses $x_{L}$ when Firm 2 chooses $x_{H}$ if $\alpha<\alpha_{b}$. Likewise, Firm 2's best response to Firm 1's choosing $x_{L}$ is $x_{L}$ if $\alpha<\alpha_{c}$ (i.e., if $\left.\alpha<\alpha_{c}, \pi_{2 \mid\left(x_{L}, x_{L}\right)}>\pi_{2 \mid\left(x_{L}, x_{H}\right)}\right)$ when $\lambda<\tilde{\lambda}$ or $\lambda \geq \hat{\lambda}$. When $\tilde{\lambda} \leq \lambda<\hat{\lambda}$, Firm 2's best response to Firm 1's choosing $x_{L}$ is $x_{L}$ in the all possible range of $\alpha$. Firm 2's best response to Firm 1's choosing $x_{H}$ is $x_{L}$ if $\alpha<\alpha_{d}$ (i.e., if $\left.\alpha<\alpha_{d}, \pi_{2 \mid\left(x_{H}, x_{L}\right)}>\pi_{2 \mid\left(x_{H}, x_{H}\right)}\right)$. Therefore, both Firms 1 and 2 choose $x_{L}$ irrespective of the competitor's decision when $\alpha<\bar{\alpha}\left(=\min \left(\alpha_{a}, \alpha_{b}, \alpha_{c}, \alpha_{d}\right)\right) . \alpha_{a}, \alpha_{b}, \alpha_{c}$ and $\alpha_{d}$ are derived from the equations $\pi_{1 \mid\left(x_{L}, x_{L}\right)}=\pi_{1 \mid\left(x_{H}, x_{L}\right)}$,

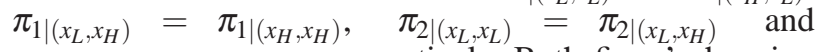
$\pi_{2 \mid\left(x_{H}, x_{L}\right)}=\pi_{2 \mid\left(x_{H}, x_{H}\right)}$, respectively. Both firms' choosing $x_{L}$ is the unique subgame-perfect Nash equilibrium when $\alpha<\bar{\alpha}$. Here, $\bar{\alpha}=\alpha_{c}$ when $\lambda<\tilde{\lambda}, \bar{\alpha}=\alpha_{b}$ when $\tilde{\lambda} \leq \lambda<\dot{\lambda}, \bar{\alpha}=\alpha_{a}$ when $\dot{\lambda} \leq \lambda<\hat{\lambda}$, and $\bar{\alpha}=\alpha_{c}$ when $\lambda \geq \hat{\lambda}$.
Proposition 2. When the gap in firms' ability to utilize user information is large $(\lambda<\tilde{\lambda})$, and many users are privacy seekers (i.e., when the portion of convenience seekers is in the range of $\bar{\alpha}<\alpha \leq \tilde{\alpha})$, the firm with the superior ability chooses to demand basic information $\left(x_{L}\right)$ from users to serve both types of users including privacy seekers and convenience seekers while the firm with the inferior ability chooses to demand highly private information $\left(x_{H}\right)$ from users only to serve convenience seekers. That is, $\left(x_{L}, x_{H}\right)$ is the unique subgame-perfect Nash equilibrium under this condition.

Proof. Firm 1 chooses $x_{L}$ irrespective of Firm 2's decision when $\alpha<\tilde{\alpha}\left(=\min \left(\alpha_{a}, \alpha_{b}\right)\right)$. When $\lambda<\tilde{\lambda}, \bar{\alpha}=\alpha_{c}$ and $\alpha_{c}<\tilde{\alpha}$. In the range of $\bar{\alpha} \leq \alpha<\tilde{\alpha}$, Firm 1 chooses $x_{L}$ irrespective of Firm 2's decision. Firm 2's best response to Firm 1's choosing $x_{L}$ is $x_{H}$. Therefore, Firm 1's choosing $x_{L}$ and Firm 2's choosing $x_{H}$ is the unique subgame-perfect Nash equilibrium under the condition of $\lambda<\tilde{\lambda}$ and $\bar{\alpha} \leq$ $\alpha<\tilde{\alpha}$.

Under the condition of $\lambda<\tilde{\lambda}$ and $\bar{\alpha} \leq \alpha<\tilde{\alpha}$, Firm 1 (the firm with the superior ability) chooses $x_{L}$ irrespective of Firm 2's (the firm with the inferior ability) choice of the level of information demanded of users. When Firm 1 chooses $x_{L}$, Firm 2's best response is to choose $x_{H}$. Therefore, at equilibrium, Firm 1 chooses $x_{L}$, and Firm 2 chooses $x_{H}$.

Firm 1 chooses $x_{L}$ irrespective of Firm 2's decision because many users are privacy seekers. However, unlike Firm 1, Firm 2 chooses $x_{H}$ instead of $x_{L}$. As noted above, when Firm 2 chooses $x_{H}$, it cannot attract privacy seekers. However, in choosing $x_{H}$, Firm 2 can increase its service quality (still $q_{1}>q_{2}$, since $\lambda<\tilde{\lambda}$ ) and charge higher prices to convenience seekers. Under the condition noted in this proposition, the benefit from the latter overcomes the loss from the former. Therefore, the firm can generate greater profit by choosing $x_{H}$ rather than by choosing $x_{L}$. The figure 1 shows how the price, market share, and profit of Firm 2 are affected by Firm 2 choosing $x_{H}$ instead of $x_{L}$ when Firm 1 chooses $x_{L}$ under the condition of $\lambda=0.3(<\tilde{\lambda})$ and $\bar{\alpha}<\alpha \leq \tilde{\alpha}$.

Proposition 3. When the gap in firms' ability to utilize customer information is in the range of $\tilde{\lambda} \leq \lambda<\hat{\lambda}$, and many users are privacy seekers (i.e., when the portion of convenience seekers is in the range of $\bar{\alpha}<\alpha \leq \tilde{\alpha}$ ), both firms choose to demand basic information $\left(x_{L}\right)$ from users to serve both types of users including convenience seekers and privacy seekers. That is, $\left(x_{L}, x_{L}\right)$ is the unique subgame-perfect Nash equilibrium under this condition.

Proof. When $\tilde{\lambda} \leq \lambda<\hat{\lambda}, \pi_{2 \mid\left(x_{L}, x_{L}\right)}>\pi_{2 \mid\left(x_{L}, x_{H}\right)}$ in all possible ranges of $\alpha$. Therefore, whenever Firm 1 chooses $x_{L}$, Firm 2 choose $x_{L}$. Therefore, $\left(x_{L}, x_{L}\right)$ is the unique subgame-perfect Nash equilibrium under the condition of $\tilde{\lambda} \leq \lambda<\hat{\lambda}$ and $\bar{\alpha} \leq \alpha<\tilde{\alpha}$.

When $\tilde{\lambda} \leq \bar{\lambda}<\hat{\lambda}$, Firm 2 choosing $x_{H}$ when Firm 1 chooses $x_{L}$ results in similar quality service from both firms. This intensifies competition between the firms. 


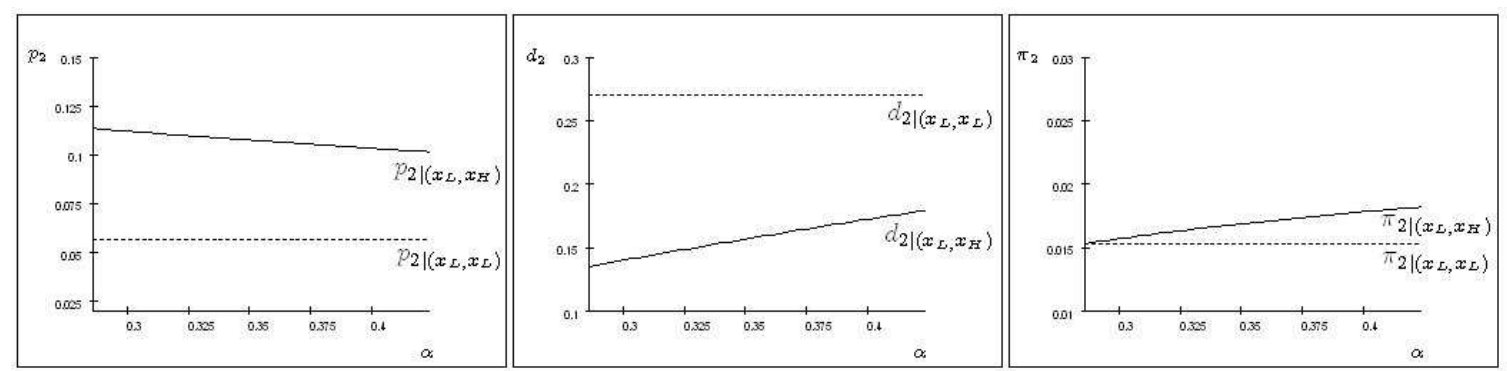

Fig. 1: Price, market share, and profit of Firm 2 when choosing $x_{H}$ or $x_{L}$ when Firm 1 chooses $x_{L}$ under the condition of $\lambda=0.3$

That is why the equilibrium is different from that of the previous proposition. The figures 2 show how Firm 2 can be worse off choosing $x_{H}$ when Firm 1 chooses $x_{L}$ when $\lambda=0.47(\tilde{\lambda}<0.47<\hat{\lambda})$. Firm 2's choice of $x_{H}$ is dominated by the firm's choice of $x_{L}$. Therefore, unlike the case in proposition 2, Firm 2 has no incentive to choose $x_{H}$ when Firm 1 chooses $x_{L}$ under the condition of $\tilde{\lambda} \leq \lambda<\hat{\lambda}$. Hence, both firms choosing $x_{L}$ results in equilibrium under this condition.

Proposition 4. When the gap in the firms' ability to utilize user information is small $(\lambda \geq \hat{\lambda})$, and many users are privacy seekers (i.e., when the portion of convenience seekers is in the range of $\bar{\alpha}<\alpha \leq \tilde{\alpha})$, the firm with the superior ability chooses to demand basic information $\left(x_{L}\right)$ from users to serve both types of users including privacy seekers and convenience seekers, while the firm with the inferior ability chooses to demand highly private information $\left(x_{H}\right)$ from users only to serve convenience seekers. That is, $\left(x_{L}, x_{H}\right)$ is the unique subgame-perfect Nash equilibrium under this condition.

Proof. When $\lambda \geq \hat{\lambda}, \bar{\alpha}=\alpha_{c}$ and $\alpha_{c}<\tilde{\alpha}$. In the range of $\bar{\alpha} \leq \alpha<\tilde{\alpha}$, Firm 1 chooses $x_{L}$ irrespective of Firm 2's decision, and Firm 2's best response is $x_{H}$. Therefore, Firm 1 's choosing $x_{L}$ and Firm 2's choosing $x_{H}$ is the unique subgame-perfect Nash equilibrium under the condition of $\lambda \geq \hat{\lambda}$ and $\bar{\alpha} \leq \alpha<\tilde{\alpha}$.

Under the condition of $\lambda \geq \hat{\lambda}$ and $\bar{\alpha}<\alpha \leq \tilde{\alpha}$, Firm 1 chooses $x_{L}$ irrespective of Firm 2's decision to meet many users' privacy concerns. In this case, Firm 2 choosing $x_{H}$ when Firm 1 chooses $x_{L}$ enables Firm 2 to provide its superior quality service to convenience seekers, compared to that of Firm 1 (i.e., $q_{2}>q_{1}$, where the gap between $q_{2}$ and $q_{1}$ is not too small). Therefore, Firm 2 can charge a higher price for the firm's service. Even though the firm cannot attract privacy seekers, the firm can generate greater profit from convenience seekers by choosing $x_{H}$ instead of $x_{L}$. The figure 3 shows how the price, market share, and profit of Firm 2 are affected by Firm 2 choosing $x_{H}$ instead of $x_{L}$ when Firm 1 chooses $x_{L}$ under the condition of $\lambda=0.6(>\hat{\lambda})$ and $\bar{\alpha}<\alpha \leq \tilde{\alpha}$.

Proposition 5. When the gap in firms' ability to utilize user information is small (i.e., $\lambda \geq \breve{\lambda}(>\hat{\lambda})$ ), the profit of the firm with the inferior ability at equilibrium $\left(x_{L}, x_{H}\right)$ under conditions when the portion of convenience seekers is in the range of $\breve{\alpha}<\alpha \leq \tilde{\alpha}$ is greater than that of the firm at equilibrium $\left(x_{H}, x_{H}\right)$ in the absence of privacy seekers $(\alpha=1)$.

Proof. Under the condition of $\lambda \geq \breve{\lambda}(>\hat{\lambda})$, the profit of Firm 2 at equilibrium $\left(x_{H}, x_{H}\right)$ when $\alpha=1$ is $\pi_{2 \mid\left(x_{H}, x_{H}\right), \alpha=1}=2 \lambda(1-\lambda) /\left(\lambda^{2}-8 \lambda+16\right)$.

When we compare $\pi_{2 \mid\left(x_{H}, x_{H}\right), \alpha=1}$ with the profit of Firm 2 at equilibrium $\left(x_{L}, x_{H}\right)$ where $\bar{\alpha}<\alpha \leq \tilde{\alpha}$, $\pi_{2 \mid\left(x_{L}, x_{H}\right)}, \pi_{2 \mid\left(x_{L}, x_{H}\right)}>\pi_{2 \mid\left(x_{H}, x_{H}\right), \alpha=1}$ in the range of $\alpha$, $\breve{\alpha}<\alpha \leq \tilde{\alpha}$. Here, $\breve{\alpha}>\bar{\alpha}$. $\breve{\alpha}$ is derived from the equation $\pi_{2 \mid\left(x_{L}, x_{H}\right)}=\pi_{2 \mid\left(x_{H}, x_{H}\right), \alpha=1}$.

We can infer from this proposition that serious user concern about information privacy is not always bad for
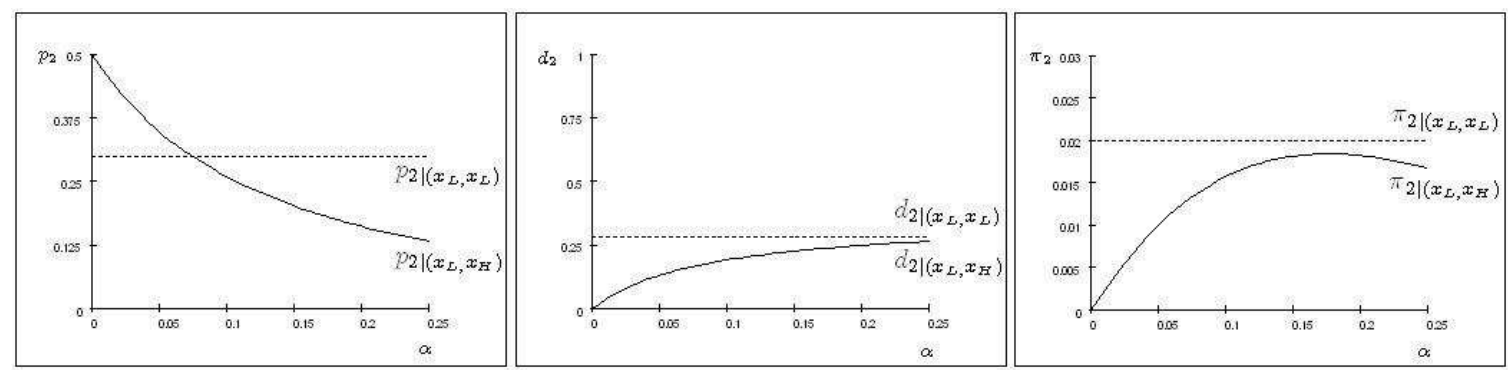

Fig. 2: Price, market share, and profit of Firm 2 when choosing $x_{H}$ or $x_{L}$ when Firm 1 chooses $x_{L}$ under the condition of $\lambda=0.47$ 

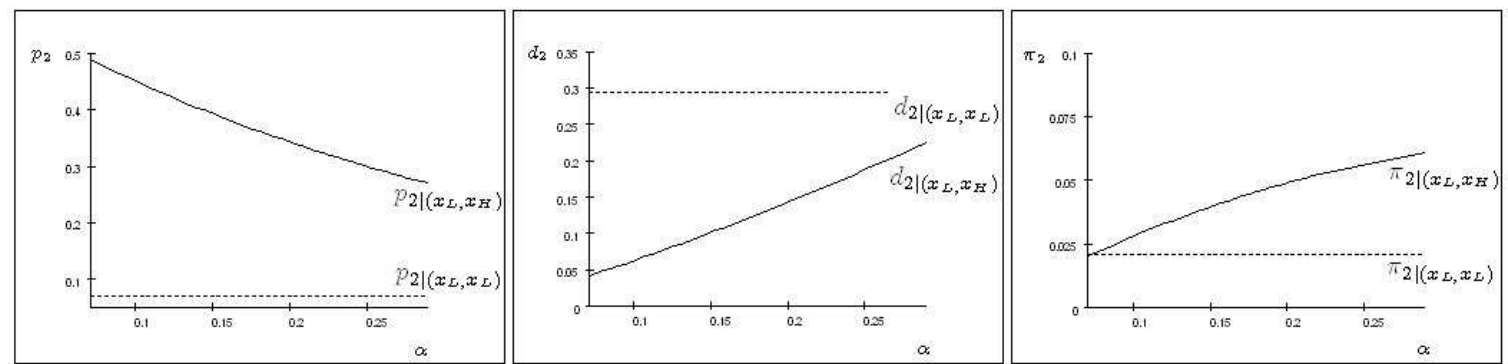

Fig. 3: Price, market share, and profit of Firm 2 when choosing $x_{H}$ or $x_{L}$ when Firm 1 chooses $x_{L}$ under the condition of $\lambda=0.6$

firms. In the absence of privacy seekers $(\alpha=1)$, both firms choose $x_{H}$ at equilibrium. However, in the presence of high levels of privacy concern $(\breve{\alpha}<\alpha \leq \tilde{\alpha})$ under the condition of $\lambda \geq \breve{\lambda}(>\hat{\lambda})$, Firm 1 chooses $x_{L}$ and Firm 2 chooses $x_{H}$ at equilibrium. In this case, even though Firm 2 cannot attract privacy seekers, it can charge higher prices to convenience seekers by providing superior service compared to that of Firm 1. This provides Firm 2 with an opportunity to increase the firm's profit. The figure 4 compares the profit of Firm 2 at equilibrium $\left(x_{L}\right.$, $\left.x_{H}\right)$ under the condition of $\lambda=0.72(>\breve{\lambda})$ and $(\breve{\alpha}<\alpha \leq \tilde{\alpha})$ and that of Firm 2 at equilibrium $\left(x_{H}, x_{H}\right)$ under the condition of $\lambda=0.72$ and $\alpha=1$. The figure shows that the profit under conditions of privacy concern clearly dominates that without it.

Proposition 6. When the gap in firms' ability to utilize user information is small (i.e., $\lambda \geq \bar{\lambda}(>\hat{\lambda})$ ), the profit of the firm with superior ability at equilibrium $\left(x_{L}, x_{H}\right)$ in the presence of the existence of many privacy seekers $(\bar{\alpha}<$ $\alpha \leq \widehat{\alpha})$ is greater than that of the firm at equilibrium $\left(x_{H}\right.$, $\left.x_{H}\right)$ in the absence of privacy seekers $(\alpha=1)$.

Proof. When $\lambda \geq \bar{\lambda}(>\hat{\lambda})$ and $\alpha=1$, the profit of Firm 1 at equilibrium $\left(x_{H}, x_{H}\right)$ is $\pi_{1 \mid\left(x_{H}, x_{H}\right), \alpha=1}=8(1-\lambda) /\left(\lambda^{2}-8 \lambda+16\right)$.

When we compare $\pi_{1 \mid\left(x_{H}, x_{H}\right), \alpha=1}$ with the profit of Firm 1 at equilibrium $\left(x_{L}, x_{H}\right)$ where $\bar{\alpha}<\alpha \leq \tilde{\alpha}$, $\pi_{1 \mid\left(x_{L}, x_{H}\right)}, \pi_{1 \mid\left(x_{L}, x_{H}\right)} \geq \pi_{1 \mid\left(x_{H}, x_{H}\right), \alpha=1}$ in the range of $\alpha$, $\bar{\alpha}<\alpha \leq \widehat{\alpha}$ (here, $\hat{\alpha}<\tilde{\alpha}$ ). $\widehat{\alpha}$ is derived from the equation $\pi_{1 \mid\left(x_{L}, x_{H}\right)}=\pi_{1 \mid\left(x_{H}, x_{H}\right), \alpha=1}$.

\section{Conclusions}

Managing users' privacy concern is an important issue for cloud service firms. In this study, considering the heterogeneity of users in their privacy concern, we show how firms should design their cloud service in terms of privacy. We show that firms' differing strategy in designing their service in terms of privacy is required especially when the portion of privacy sensitive users is not small. We also show that privacy concerns are not just a barrier to be overcome but could be strategic resources for market segmentation if well managed. This fact also implies that privacy issues may be handled to a certain extent with market mechanisms rather than direct legal measures originating from the government.

However, there are some limitations of our research. For the tractability of our analytical model, we simplified the real world and abstracted cloud service system as a black box. These and other realistic conditions add much more complexity to firms' decisions in implementing a cloud service and handling customer information. Therefore, further empirical research or practical reports are desired.

The authors are grateful to the anonymous referee for a careful checking of the details and for helpful comments that improved this paper.

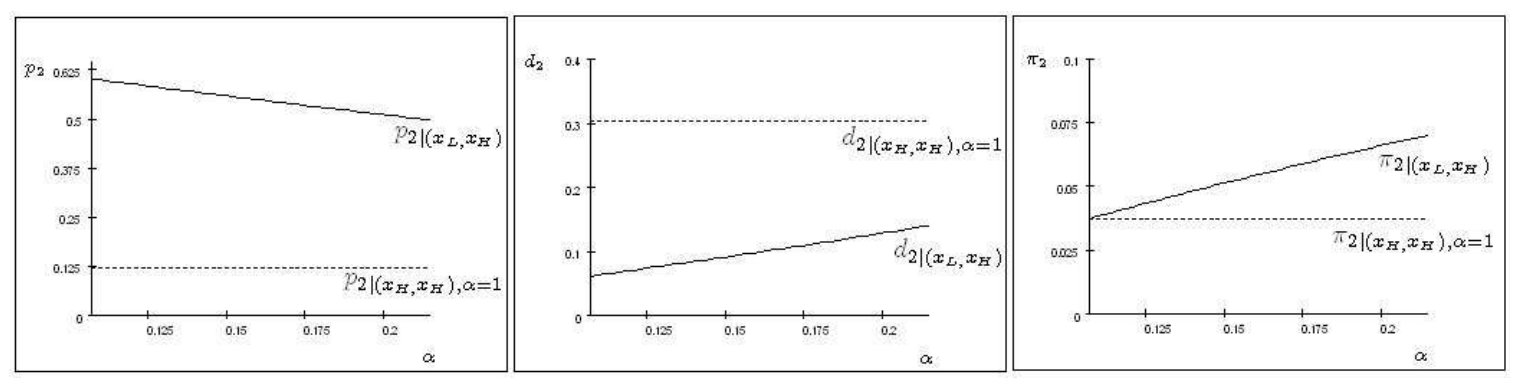

Fig. 4: Price, market share, and profit of Firm 2 at equilibrium when $\lambda=0.72$ 


\section{References}

[1] A. Acquisti, Security of personal information and privacy: Technological solutions and economic incentives, The Economics of Information Security, Kluwer, (2004).

[2] Culnan, Mary J, Protecting privacy online: Is self-regulation working?, Journal of Public Policy \& Marketing, 19, 20 (2000).

[3] Derlega, Valerian J, Metts Sandra, Petronio Sandra and Margulis, Stephen T, Self-disclosure, Sage Publications, Inc., (1993).

[4] Chellappa, Ramnath K and Sin, Raymond G, Personalization versus privacy: An empirical examination of the online consumers dilemma, Information Technology and Management, 6, 181 (2005).

[5] Chellappa, Ramnath K and Shivendu Shivendu, An economic model of privacy: A property rights approach to regulatory choices for online personalization, Journal of Management Information Systems, 24, 193 (2007).

[6] D. Fudenberg and J. Tirole, Game Theory, Cambridge, MA: MIT Press, (1991).

[7] Payton F. C, Ecommerce: Technologies That Do Steal!, Decision Line, 13 (2001).

[8] Allard C. R. Riel, Liljander Veronica and Jurriens Petra, Exploring consumer evaluations of e-services: a portal site, International Journal of Service Industry Management, 12, 359 (2001).

[9] Rust, Roland T, Zahorik, Anthony J and Keiningham, Timothy L, Return on quality (ROQ): making service quality financially accountable, The Journal of Marketing, 59, 58 (1995).

[10] Ruyter Ko de, Wetzels Martin and Kleijnen Mirella, Customer adoption of e-service: an experimental study, International Journal of Service Industry Management, 12, 184 (2001).

[11] Zeithaml, Valarie A. Berry, Leonard L and Parasuraman A, The behavioral consequences of service quality, The Journal of Marketing, 60, 31 (1996).

[12] Zeithaml, Valarie A. Rust, Roland T and Lemon, Katherine $\mathrm{N}$, The customer pyramid: creating and serving profitable customers, California Management Review, 43, 118 (2001).

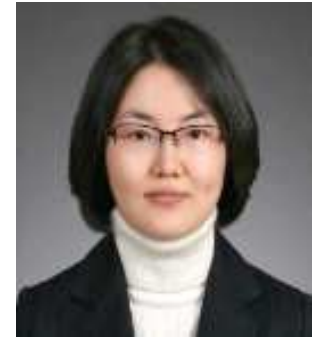

Eunjin Kim received her B.S., M.S. and Ph.D degrees in Management from KAIST. She is an assistant professor at Kyonggi University. Her current research interests include economic analysis of digital content, information systems and effects of the digital divide. 